\title{
Stress Stiffening and Approximate Equations in Flexible Multibody Dynamics
}

\author{
Carlos E. Padilla* \\ Photon Research Associates, Inc. \\ Cambridge, Massachusetts 02138 \\ and \\ Andreas H. von Flotow ${ }^{\dagger}$ \\ Massachusetts Institute of Technology \\ Cambridge, Massachusetts 02139
}

\begin{abstract}
A useful model for open chains of flexible bodies undergoing large rigid body motions, but small elastic deformations, is one in which the equations of motion are linearized in the small elastic deformations and deformation rates. For slow rigid body motions, the correctly linearized, or consistent, set of equations can be compared to prematurely linearized, or inconsistent, equations and to "oversimplified," or ruthless, equations through the use of open loop dynamic simulations. It has been shown that the inconsistent model should never be used, while the ruthless model should be used whenever possible. The consistent and inconsistent models differ by stress stiffening terms. These are due to zeroth-order stresses effecting virtual work via nonlinear strain-displacement terms. In this paper we examine in detail the nature of these stress stiffening terms and conclude that they are significant only when the associated zeroth-order stresses approach "buckling" stresses. Finally it is emphasized that when the stress stiffening terms are negligible the ruthlessly linearized equations should be used.
\end{abstract}

\section{Introduction}

In a previous paper ${ }^{1}$ it was suggested that a useful model for open chains of flexible bodies undergoing large rigid body motions, but small elastic deformations, would be one in which the equations of motion are linearized in the small elastic deformations and deformation rates. In that paper, it was pointed out that in order to obtain a consistently linearized set of equations of motion it is necessary to make use of nonlinear strain-

\footnotetext{
* Staff Scientist, Dynamics and Control Division.

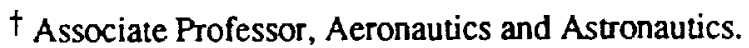


displacement relations, ${ }^{2.3}$ nonlinear kinematic constraints, 4,5 or several nonlinear geometric or motion stiffness terms appended to the incorrectly linearized equations of motion. ${ }^{6.7}$ It is easy to verify that equations of this type, linearized in only some of the states, do not conserve energy.

A presumed goal of simplified equations of motion is to obtain accurate simulation of actual system behavior with a minimum of effort. At present, there is no standardized yardstick with which to determine how various simplified equations measure up. It has been suggested ${ }^{14}$ that those simplified equations which come closest to conserving energy should be considered most appropriate. In that paper, it is shown how equations that contain second order terms in the flexible states can be obtained from prematurely linearized velocity expressions. 4,5 But these equations, while perfectly conserving energy, still lack the first order terms (stress stiffening terms) that correctly model the physical nature of the system! In light of this example it becomes clear that conservation of energy, while desirable for numerical reasons, is not a good measure of the adequacy of a simplified set of equations of motion.

Equations in which all nonlinear terms involving the flexible generalized coordinates and their time rates of change are ignored have been termed "ruthlessly linearized".1 The correctly linearized, or consistent, set of equations was compared to prematurely linearized, or inconsistent, equations and to the ruthless equations by means of open loop dynamics simulations for the case of slow rigid body motions. It was concluded that the inconsistent model should never be used, while the ruthless model should be used whenever possible. We point out that the ruthless model conserves energy. This stems from the fact that the ruthless model can be derived from a set of velocity expressions without any further simplifications, i.e., no terms are dropped after forming the velocity expressions. This ensures that the mass matrix remains positive definite for all possible trajectories.

The inconsistent model results from neglecting certain kinematic relationships between the elastic deformation variables, or what is equivalent, to using linear straindisplacement relations. This results in the absence of certain terms linear in the elastic coordinates and rates. The missing terms have been identified as the so-called geometric stiffness terms of certain rotational dynamics problems. These terms are also known as stress or motion stiffness terms and are not limited to rotational forces. They result from a combination of zeroth-order stresses and nonlinear strain-displacement relations in the virtual energy expressions that result in terms linear in the strain (displacement) variables. ${ }^{10,7.9}$

In the rest of the paper we consider the nature of the stress stiffening terms and their role in the determination of simplified equations of motion. In section II we present the general form of equations of motion for open kinematic chains of flexible bodies. The ruthless model is then defined and it is shown how this model can be derived directly. In section III we consider stress stiffness terms: their derivation; the forces that give rise to them; and their general form. An analytical example is provided using a two link flexible manipulator. Finally, in section IV we consider the importance of these stress stiffening terms for the formulation of equations of motion. 


\section{Equations of Motion for Chains of Elastic Bodies}

The equations of motion of an open chain of elastic bodies can be expressed quite generally as: ${ }^{8}$

$$
\begin{gathered}
{\left[\begin{array}{cc}
M_{R R}(x, q) & M_{R E}(x, q) \\
M_{E R}(x, q) & M_{E E}(x, q)
\end{array}\right]\left[\begin{array}{l}
\dot{x} \\
\dot{q}
\end{array}\right]+\left[\begin{array}{ll}
0 & 0 \\
0 & K_{E E}
\end{array}\right]\left[\begin{array}{l}
x \\
q
\end{array}\right]=\left[\begin{array}{c}
T_{\text {ext }, R} \\
T_{\text {ext }, E}
\end{array}\right]+\left[\begin{array}{c}
F_{R}(x, q, \dot{x}, u) \\
F_{E}\left(x, q, x^{\prime}, u\right)
\end{array}\right]} \\
u=\dot{q}
\end{gathered}
$$

where $x$ is a vector of rigid body generalized coordinates; $q$ is a vector of the elastic generalized coordinates; $M_{R R}, M_{R E}, M_{E R}, M_{E E}$ form the configuration-dependent mass matrix; $T_{e x t}$ is a vector of generalized extemal forces; $K_{E E}$ is a constant stiffness matrix and $F$ is a vector of nonlinear inertial (coriolis and centripetal) forces. Subscripts $R$ and $E$ denote rigid and elastic, respectively.

We are often interested in the important class of systems for which the elastic deformations remain small so we can ignore terms of second and higher order in $q$ and $u$. In this case, Eq. (1) can be expanded to show more explicitly the form of the nonlinear terms:

$$
\begin{aligned}
& {\left[\begin{array}{cc}
M_{R R}(x, q) & M_{R E}(x) \\
M_{E R}(x) & M_{E E}(x)
\end{array}\right]\left[\begin{array}{l}
\dot{x} \\
\dot{q}
\end{array}\right]+\left[\begin{array}{ll}
0 & 0 \\
0 & K_{E E}
\end{array}\right]\left[\begin{array}{l}
x \\
q
\end{array}\right]=\left[\begin{array}{c}
T_{e x t, R} \\
T_{e x t, E}
\end{array}\right]+\left[\begin{array}{l}
F_{R 0}\left(x, x^{\prime}\right) \\
F_{E 0^{\prime}}\left(x, x^{*}\right)
\end{array}\right]} \\
& +\left[\begin{array}{c}
F_{R 1}\left(x, x^{*}\right) \\
F_{E 1}^{*}\left(x, x^{*}, x^{*}\right)
\end{array}\right] q+\left[\begin{array}{c}
F_{R 2}\left(x, x^{*}\right) \\
F_{E 2}\left(x, x^{*}\right)
\end{array}\right] \dot{q}
\end{aligned}
$$

Notice in the above equation that we have lumped the terms in $M_{E R}$ that depend on $q$ together with the $F_{E I}$ term and have denoted the new term by $F_{E I} I^{*}$.

In Eq. (2), the superscript * terms are the only terms that could contain foreshortening terms, i.e., terms arising from the enforcement of kinematic constraints among the flexible degrees of freedom of the elastic bodies. These terms, which can be obtained equivalently by the use of nonlinear strain-displacement relations in the formation of the inertial velocities of the bodies, are missing when linear strain-displacement relations are used instead, i.e., when the equations are linearized prematurely. 5.6 In the case of a single flexible body, the incorrectly linearized equations can then be fixed through the use of "motion stiffness" matrices. 7 


\section{II.A The Ruthlessly Linearized Model}

In a "ruthlessly linearized" model, ${ }^{1}$ we simplify the equations of motion for a chain of flexible bodies by ignoring all nonlinear terms that involve the elastic generalized coordinates and rates $(q$ and $u)$, including those terms in the mass matrix which depend on elastic coordinates. In this case Eq. (2) becomes:

$$
\left[\begin{array}{cc}
M_{R R}(x) & M_{R E}(x) \\
M_{E R}(x) & M_{E E}(x)
\end{array}\right]\left[\begin{array}{c}
\dot{x} \\
\dot{q}
\end{array}\right]+\left[\begin{array}{ll}
0 & 0 \\
0 & K_{E E}
\end{array}\right]\left[\begin{array}{l}
x \\
q
\end{array}\right]=\left[\begin{array}{c}
T_{e x t, R} \\
T_{\text {ext } E}
\end{array}\right]+\left[\begin{array}{c}
F_{R 0}\left(x, x^{*}\right) \\
F_{E 0}\left(x, x^{\prime}\right)
\end{array}\right]
$$

As explained in Ref. 1, the ruthless model is a simplification of the consistently linearized equations of motion motivated in part by the so-called rate-linear assumption. This assumption consists in neglecting (coriolis and centripetal) terms nonlinear in the generalized rates, $\mathrm{d} x / \mathrm{dt}$ and $u$, for slow motion of the system. In the case of $\mathrm{n}$-link rigid manipulators it has been pointed out 12 that the velocity and acceleration terms of the dynamic equations have the same relative significance at any speed of movement. This indicates that the rate-linear assumption might not be a good one. On the other hand, terms that are nonlinear in the rigid generalized rates and linear in $q$ and $u$ might be negligible for small $q$ and $u$ when compared to similar terms that are constant in $q$ and $u$.

Whereas the consistently linearized equations of motion are theoretically valid for chains of flexible bodies undergoing fast rigid body motions, but small elastic deformations, the ruthless equations can be said to be valid for chains of flexible bodies undergoing slow rigid body motions and small elastic deformations. How slow these rigid body motions must be is the subject of section IV. Notice that both models can accommodate large configuration changes (kinematic nonlinearities), and that the distinction being made concerns only the magnitudes of the time rates of change of the rigid body generalized coordinates. In the next section, we examine how the ruthless equations of motion can be obtained without having to start with the consistent equations. Before proceeding, however, let us make some clarifications.

Up to this point, we have made little distinction between "rigid body motions" and rigid generalized coordinates and speeds. In fact, what is meant by rigid generalized speeds is the collection of generalized speeds that for each body characterize the motion of a frame of reference from which the small elastic deformations are defined. The requirement of small elastic deformations implicitly defines the frame. ${ }^{2}$ More explicit frame definitions can be made and the reader is referred to Ref. 13 for some examples. The point being made here is that for chains of flexible bodies, the so-called rigid generalized speeds do not necessarily correspond to rigid body motions, in the sense of a rigid body mode; and further whether there is a correspondence or not will depend on the choice of reference frame from which the small elastic deformations are described. This will be investigated further in section $V$ when we look at further simplifications of the ruthless model. 


\section{II.B Direct Derivation of Ruthlessly Linearized Motion Equations}

We are interested in finding a way to obtain ruthless equations without having to start with consistent equations. From the form of Eq. (3), we see that what is needed is some way of obtaining the mass matrix and the vector of coriolis and centripetal terms such that there is no $q$-dependence or $\mathrm{d} q / \mathrm{dt}$-dependence. Clearly, this could be done formally by first obtaining the consistent equations and then dropping all terms containing $q$ and $\mathrm{d} q / \mathrm{dt}$. Due to the simplicity of the resulting equations, however, it seems that there should be an easier way of obtaining Eq. (3). There is such a way, and this is in fact one of the advantages of using ruthlessly linearized equations of motion. In the following we illustrate how to attain ruthless equations for a collection of rigid and flexible bodies in a tree topology with no closed loops.

The method is very straightforward and enables the dynamicist or control designer to obtain the ruthless equations of motion for modest chains (as in, say, manipulator applications) analytically. First, form the inertial velocities of the mass centers of the rigid bodies, of points of application of external loads, and of characteristic material particles of flexible bodies. These velocities should be constant in flexible generalized coordinates, though they must be linear in generalized speeds if there are to be any flexible equations of motion. Next, form the partial velocities with respect to the generalized speeds. ${ }^{11}$ Note that the term partial velocities, coined by Kane, is a convenient way to describe partial differentiation of the velocities with respect to generalized speeds, but its use here in no way indicates that these results are exclusive to Kane's method. ${ }^{11}$

The partial velocities should now be constant in both $q$ and $\mathrm{d} q / \mathrm{dt}$, and we are ready to form the mass matrix:

$$
M_{i j}(x)=\sum_{k=1}^{N} f_{k} \mathbf{v}_{i}^{k}(x) \cdot \mathbf{v}_{j}^{k}(x) d m
$$

where the subscripts $i, j$ range over all degrees of freedom, rigid and elastic; $N$ is the number of bodies in the chain; $V_{k}$ is the volume of the $k$-th body; and $v_{l}^{k}(x)$ represents the $i$-th partial velocity of a material particle in body $k$.

In order to easily obtain the nonlinear velocity terms, we first define a pseudoacceleration. Form the acceleration for each of the inertial velocities previously defined. Note that for purposes of forming the acceleration, the inertial velocities can also be made constant in $\mathrm{d} q / \mathrm{dt}$. Once these accelerations are formed, further simplify them by dropping all terms that contain the second time derivative of any generalized coordinate or the first time derivative of the generalized speeds (since these terms have already been included in the mass matrix above). The coriolis and centripetal terms are now obtained by dot multiplying the partial velocities with the corresponding pseudo-accelerations, $\hat{a}^{k}$ : 


$$
F_{r}\left(x, x^{*}\right)=\sum_{k=1}^{N} \bigvee_{k} \mathbf{v}_{r}^{k} \cdot \mathbf{a}^{k} d m
$$

where the subscript $r$ ranges over all degrees of freedom, and $F$ is the vector of nonlinear forces in Eq. (3).

The stiffness matrix can be obtained in a variety of standard ways. ${ }^{5,10}$ Determination of the load distribution matrix is achieved as in Kane's method by dot multiplying external loads by the partial velocities (constant in $\mathrm{q}$ and $\mathrm{dq} / \mathrm{dt}$ ) of their points of application.

\section{Nonlinear Strain-Displacement and Stress Stiffness}

Enforcing geometric constraints on the generalized coordinates 5 is equivalent to retaining nonlinear strain-displacement terms in a continuum mechanics formulation of the equations of equilibrium. ${ }^{9}$ It is for this reason that appending geometric stiffness matrices 6.7 to the incorrectly linearized equations of motion yields identical results, if done consistently, to proper linearization through consideration of nonlinear geometric constraints on flexible degrees of freedom.

As defined by Ref. 10, geometric stiffness terms are used to account for second order terms in the energy expressions that result in first order effects in the motion equations but which are ignored in a premature linearization formulation. In the context of the finite element method, stresses resulting from all applied external forces are found and the virtual work they effect through the nonlinear terms of the strain expression is found to contribute linear stiffness terms that are critical in stability analysis. This has been recognized by users of finite element codes for some time, in particular as it relates to the inertial forces impressed on rotor blades by "constant" spin rates. The fact that all external, internal, and inertial forces should be taken into account when obtaining the stress resultants has perhaps not been made clear in all engineering applications. While some or all of these forces might be unimportant for particular applications, it remains true that for general, particularly dynamics, applications all forces should be considered.

In their paper, for the case of the free motion of a single flexible body, Banerjee and Dickens (Ref. 7) consider only forces due to impressed motions, or inertial forces. They extend the concept of geometric or stress stiffness matrices by considering time-varying inertial forces. This is achieved (see also Ref. 9) by obtaining the geometric stiffness matrices assuming unit values of a set of 21 inertia loads taken one at a time and using a standard finite element code. Invoking linearity, they then proceed to multiply each motion stiffness matrix by the instantaneous value of the associated inertia load and to add the resulting matrices together at each instant in time.

The above procedure results in an additional stiffness term being added to the flexible partition of the inconsistently linearized equations (Eq. (2) with the superscript * terms lacking foreshortening terms): 


$$
K_{g} q=-\sum_{k=1}^{2} S^{(k)} A_{k} q
$$

$K_{g}$ above is the generalized motion stiffness for a single flexible body and $S^{(k)}$ is the geometric stiffness matrix corresponding to unit values of the $k$-th inertia loading, $A_{k}$, with the other inertia loadings set to zero. The $A_{k}$ in Eq. (6) are defined as follows: ${ }^{7}$

$$
\begin{array}{llll}
A_{1}=u_{1}+u_{5} u_{3}-u_{6} u_{2} & A_{4}=-\left(u_{5}^{2}+u_{6}^{2}\right) & A_{7}=-u_{6}+u_{4} u_{5} & A_{10}=u_{5}+u_{6} u_{4} \\
A_{2}=u_{2}+u_{6} u_{1}-u_{4} u_{3} & A_{5}=u_{6}+u_{4} u_{5} & A_{8}=-\left(u_{6}^{2}+u_{4}^{2}\right) & A_{11}=-u_{4}+u_{5} u_{6} \\
A_{3}=u_{3}+u_{4} u_{2}-u_{5} u_{1} & A_{6}=-u_{5}+u_{6} u_{4} & A_{9}=u_{4}+u_{5} u_{6} & A_{12}=-\left(u_{5}^{2}+u_{4}^{2}\right)
\end{array}
$$

where for simplicity we have ignored rotary inertia effects in the flexible body, thus setting $A_{k}$ for $k=13$ to 21 equal to zero (see Ref.7). The $u_{i}(i=1, \ldots, 6)$ are the generalized speeds characterizing the motion of a frame of reference attached to the body and are defined as: ${ }^{11}$

$$
\begin{aligned}
u_{i}=\mathrm{v}^{O} \cdot \mathrm{b}_{i}, & i=1,2,3 \\
u_{i+3} & =\omega^{B} \cdot \mathrm{b}_{i}, \quad i=1,2,3
\end{aligned}
$$

where $O$ is the origin of the frame $B\left(\mathbf{b}_{1}, \mathbf{b}_{2}, \mathbf{b}_{3}\right)$ attached to the flexible body; $v^{O}$ is the inertial velocity of $O$; and $\omega^{B}$ is the inertial angular velocity of $B$.

The above methods rely on a finite element analysis in which the displacement interpolation functions are nonlinear. ${ }^{19}$ This is equivalent to considering nonlinear straindisplacement relations in a continuum formulation. Notice that a proper finite element code is capable of generating the correct results (i.e., a formulation of the equations of motion that contains all the motion stiffness terms) without any tampering, but at a high computational cost (see, e.g., Ref. 15). The method of Banerjee and Dickens can be likened to a preprocessing of the equations so that motion stiffness matrices are computed only once. This yields large savings in computational cost.

It is clear that a similar process is viable for all loadings that generate zeroth-order stresses, including joint interconnection forces for chains of flexible bodies. Wallrap and Schwertassek ${ }^{15}$ show how this can be done for each body in a chain. Their method requires knowledge of interconnection forces at each instant in time, however, and these depend in general on acceleration terms. The motion equations thus can become implicit in the accelerations. Kim ${ }^{16}$ proposes an iterative solution approach for the interconnection forces which they claim converges in very few iteration steps at each integration time step. The 
iteration is started with some a priori guesses for the accelerations. Their method does not require updating the mass matrix at each iteration step.

\section{III.A Possible Stress Stiffness Matrices}

As mentioned earlier, all zeroth-order forces (which will result in zeroth-order stresses) can potentially generate stress stiffness matrices. Consequently, the following forces must be considered:

1) applied loads,

2) gravity loads,

3) motion-induced forces: both body inertial forces and interconnection forces.

The general form of the resulting stress stiffness matrices is given by:

$$
\left[K_{G}\right]_{B a d y k}-\int_{V_{k}}\left[\phi_{i j} \phi_{j, m}\right] \sigma_{o} d V_{k}
$$

where (.) $i, j$ represents the partial derivative in the $j$-th direction of (.) in the $i$-th direction.

\section{III.B Two-Flexible-Link Arm Example}

In Ref. 3 the consistently linearized equations of motion for a planar, revolute, twoflexible-link manipulator arm are derived. Nonlinear strain-displacement relations for a bar are used in the modelling of each link as a Bernoulli-Euler beam. The resulting equations contain all terms linear in the flexible coordinates and their time rates of change. In particular, the stress stiffness terms are included in an implicit way. In this section we rewrite these terms due to the nonlinear strain-displacement relations (also dubbed foreshortening terms) and show explicitly that they indeed possess the general form shown in the previous section.

The transverse link displacements $u_{1}$ and $u_{2}$ of the shoulder and elbow links, respectively, are discretized using an assumed modes expansion:

$$
\begin{aligned}
u_{1} & =\sum_{i=1}^{n} \phi_{i}(x) q_{i}(t) \\
u_{2} & =\sum_{i=1}^{m} \psi_{i}(y) p_{i}(t)
\end{aligned}
$$


The absolute shoulder angular position is given by $\theta$, and the relative angular position of the elbow is given by $\beta$.

For the shoulder link, two zeroth-order forces yield stress stiffness matrices. The first one is due to the body distributed centripetal load:

$$
\left[K_{G_{1}}\right]_{L_{1}}=\left[\frac{m_{1} l_{1}}{2} \int_{0}^{l_{1}} \phi_{i}^{\prime} \phi_{j}^{\prime} d x-\int_{0}^{l_{1}} \rho_{1} \frac{x^{2}}{2} \phi_{i}^{\prime} \phi_{j}^{\prime} d x\right] \dot{\theta}^{2}
$$

The second stress stiffness matrix is due to the axial component (along the neutral axis of the undeformed shoulder link) of the interconnection force at the elbow joint:

$$
\left[K_{G_{2}}\right]_{L_{1}}-\left[\int_{0}^{l_{1}} \phi_{i}^{\prime} \phi_{j}^{\prime} d \sigma\right] \frac{1}{2} m_{2} l_{2}\left[\sin (\beta)(\ddot{\theta}+\ddot{\beta})+\cos (\beta)(\dot{\theta}+\dot{\beta})^{2}\right]
$$

In the above equations $\rho_{1}$ and $\rho_{2}$ are the mass per unit length of the shoulder and elbow links, respectively, and $m_{1}=\rho_{1} l_{1}, m_{2}=\rho_{2} l_{2}$ are the respective link masses.

The stress stiffness matrices for the elbow link can be considered to be due either to the inertial body forces, or to the axial component (along the neutral axis of the undeformed elbow link) of the interconnection force at the elbow joint:

$$
\begin{gathered}
{\left[K_{G_{1}}\right]_{L_{2}}-\left[\frac{m_{2} l_{2}}{2} \int_{0}^{h} \Psi_{i}^{\prime} \Psi_{j}^{\prime} d y-\int_{0}^{h} \rho_{2} \frac{y^{2}}{2} \psi_{i}^{\prime} \Psi_{j}^{\prime} d y\right](\dot{\theta}+\dot{\beta})^{2}} \\
{\left[K_{\left.G_{2}\right]_{L_{2}}}=\left[m_{2} \int_{0}^{h} \Psi_{i}^{\prime} \Psi_{j}^{\prime} d y-\int_{0}^{h} \rho_{2} y \psi_{i}^{\prime} \Psi_{j}^{\prime} d y\right] l_{1}\left[-\sin (\beta) \ddot{\theta}+\cos (\beta) \dot{\theta}^{2}\right]\right.}
\end{gathered}
$$

\section{Relative Significance of Stress Stiffening Terms}

Returning to the question of simplified equations of motion, it is natural to ask when, and if, these stress stiffening terms are important. In fact, whenever these terms are important, a consistent formulation of the equations of motion is mandatory in order to model the physical system. In this case, no further simplifications are possible, beyond the linearization in the flexible states. It was shown in a previous paper ${ }^{17}$ that ruthlessly 
linearized equations are valid (i.e., accurately model the system), precisely when these stress stiffness terms are negligible. Furthermore, it can be surmised from the detail term by term comparisons made in that paper that stress stiffness terms are significant only when the appropriate zeroth-order stress is comparable to the buckling stress, i.e., when the corresponding load approaches the buckling load for the structure. This can be surmised further by considering a static stability analysis and comparing work due to loading stresses with elastic energy (see for example Refs. 10 and 18).

\section{Conclusions}

The form of ruthlessly linearized equations of motion for open chains of flexible bodies was presented. A method was outlined that allows for the easy determination of the ruthless equations of motion for a given system. We discussed the derivation of stress stiffness terms which result from nonlinear strain-displacement relations and zeroth-order stresses. These terms are needed to obtain a consistently linearized set of equations. The form of these terms was provided and it was shown that all zeroth-order loadings, not just body inertial loadings, need to be considered when determining the stress stiffening terms. It was pointed out that these terms are significant only when the associated stresses reach the level of buckling stresses. This coincides with the previously investigated limits on the validity of the ruthless equations of motion. It is concluded that ruthlessly linearized equations of motion should be used whenever stress stiffening terms are negligible.

\section{References}

1 Padilla, C. E., and von Flotow, A. H., "Nonlinear Strain-Displacement Relations and Flexible Multibody Dynamics," Journal of Guidance, Control, and Dynamics., Vol. 15, No. 1, January-February, 1992, pp. 128-136.

${ }^{2}$ Laskin, R. A., Likins, P. W., and Longman, R. W., "Dynamical Equations of a FreeFree Beam Subject to Large Overall Motions," The Journal of the Astronautical Sciences, Vol. 31, No. 4, October-December 1983, pp. 507-527.

${ }^{3}$ Padilla, C. E., "Nonlinear Strain-Displacement Relations in the Dynamics of a Two-link Flexible Manipulator," M.S. Thesis, Dept. of Aeronautics and Astronautics, Massachusetts Institute of Technology, May 1989. Also SSL Report \# 6-89.

${ }^{4}$ Ryan, R. R., "Flexibility Modeling Methods in Multibody Dynamics," AAS Paper 87 431, presented at the AAS/AIAA Astrodynamics Specialist Conference, Kalispell, Montana, August, 1987.

${ }^{5}$ Kane, T. R., Ryan, R. R., and Banerjee, A. K., "Dynamics of a Cantilever Beam Attached to a Moving Base," Journal of Guidance, Control, and Dynamics, Vol. 10, No. 2, March-April 1987, pp. 139-151.

${ }^{6}$ Spanos, J., and Laskin, R. A., "Geometric Nonlinear Effects in Simple Rotating Systems," Proceedings of the Workshop on Multibody Simulation, JPL D-5190, Vol. 1, April 15, 1988, pp. 191-218.

${ }^{7}$ Banerjee, A. K., and Dickens, J. M., "Dynamics of an Arbitrary Flexible Body Undergoing Large Rotation and Translation with Small Vibration," AIAA Paper 89-1308, April 1989. 
${ }^{8}$ Hughes, P. C., and Sincarsin, G. B., "Dynamics of an Elastic Multibody Chain: Part A - Body Motion Equations, Part B - Global Dynamics," Dynamics and Stability of Systems, Vol. 4, Nos. 3 \& 4, 1989, pp. 209-244.

${ }^{9}$ Wallrapp, O., Santos, J., and Ryu, J. "Superposition Method for Stress Stiffening in Flexible Multibody Dynamics," The Dynamics of Flexible Structures in Space: Proceedings of the International Conference on Dynamics, 1990, pp. 233-247.

10 Cook, R. D., Concepts and Applications of Finite Element Analysis, McGraw-Hill, 1985, pp. 331-341.

${ }^{11}$ Kane, T. R., and Levinson, D. A., Dynamics: Theory and Applications, McGraw -Hill Book Company, New York, 1985.

12Hollerbach, J. M., "Dynamic Scaling of Manipulator Trajectories," Journal of Dynamic Systems, Measurement, and Control, Vol. 106, March 1984, pp. 102-106.

13Likins, P. W., "Analytical Dynamics and Nonrigid Spacecraft Simulation," NASA Technical Report 32-1593, July, 1974.

${ }^{14}$ Sharf, I., and Damaren, C., "Simulation of Flexible-Link Manipulators: Basis Functions and Nonlinear Terms in the Motion Equations," Proceedings of the IEEE Robotics and Automation Conference, Nice, France, Vol. 3, May 1992, pp. 1956-1963.

15Wallrapp, O., and "Schwertassek, R., "Representation of Geometric Stiffening in Multibody System Simulation," International Journal for Numerical Methods in Engineering, Vol. 32, 1991, pp. 1833-1850.

${ }^{16}$ Ryu, J., Kim, Sung-Soo, and Kim, Sang-Sup, "An Efficient Computational Method for Dynamic Stress Analysis of Flexible Multibody Systems," Journal of Computer and Structures, Vol. 42, No. 6, 1992, pp. 969-977.

17Padilla, C.E., and von Flotow, A.H., "Further Approximations in Flexible Multibody Dynamics," Proceedings of the 32 nd AIAA/ASME/ASCE/AHS/ASC Structures, Structural Dynamics and Materials Conference, Baltimore, MD, April, 1991.

${ }^{18}$ Timoshenko, S.P., Young, D.H., and Weaver, W., Vibration Problems in Engineering, 4th ed., Wiley and Sons, 1974.

${ }^{19}$ Bathe, K.L., Finite Element Procedures in Engineering Analysis, Prentice-Hall, Englewood Cliffs, N.J., 1982. 
